\title{
Video Article \\ Proper Positioning and Restraint of a Rat Hind Limb for Focused High Resolution Imaging of Bone Micro-architecture Using In Vivo Micro-computed Tomography
}

\author{
Amanda B. Longo ${ }^{1}$, Sandra M. Sacco ${ }^{1}$, Wendy E. Ward ${ }^{1,2}$ \\ ${ }^{1}$ Department of Kinesiology, Faculty of Applied Health Sciences, Brock University \\ ${ }^{2}$ Department of Health Sciences, Faculty of Applied Health Sciences, Brock University \\ Correspondence to: Wendy E. Ward at wward@brocku.ca
}

URL: https://www.jove.com/video/56346

DOI: doi:10.3791/56346

Keywords: Bioengineering, Issue 129, bone structure, isoflurane, rodents, scan, tibia, bone

Date Published: $11 / 22 / 2017$

Citation: Longo, A.B., Sacco, S.M., Ward, W.E. Proper Positioning and Restraint of a Rat Hind Limb for Focused High Resolution Imaging of Bone Micro-architecture Using In Vivo Micro-computed Tomography. J. Vis. Exp. (129), e56346, doi:10.3791/56346 (2017).

\section{Abstract}

The use of in vivo micro-computed tomography $(\mu \mathrm{CT})$ is a powerful tool which involves the non-destructive imaging of internal structures at high resolutions in live animal models. This allows for repeated imaging of the same rodent over time. This feature not only reduces the total number of rodents required in an experimental design and thereby reduces the inter-subject variation that can arise, but also allows researchers to assess longitudinal or life-long responses to an intervention. To acquire high quality images that can be processed and analyzed to more accurately quantify outcomes of bone micro-architecture, users of in vivo $\mu \mathrm{CT}$ scanners must properly anesthetize the rat, and position and restrain the hind limb. To do this, it is imperative that the rat be anesthetized to a level of complete relaxation, and that pedal reflexes are lost. These guidelines may be modified for each individual rat, as the rate of isoflurane metabolism can vary depending on strain and body size. Proper technique for in vivo $\mu \mathrm{CT}$ image acquisition enables accurate and consistent measurement of bone micro-architecture within and across studies.

\section{Video Link}

The video component of this article can be found at https://www.jove.com/video/56346/

\section{Introduction}

The use of in vivo micro-computed tomography $(\mu \mathrm{CT})$ is a powerful tool which involves the non-destructive imaging of internal structures at high resolutions using rodent models. The non-destructive nature of in vivo $\mu C T$ allows for repeated imaging of the same rodent over time. This feature not only reduces the total number of rodents required in an experimental design and thereby reduces the inter-subject variation that can arise, but also allows researchers to understand long-term responses to an intervention. With the use of repeated in vivo $\mu \mathrm{CT}$, experiments in mice and rats have elucidated developmental changes to bone micro-architecture and bone mineral density (BMD) throughout periods of the lifespan ${ }^{1,2,3,4,5,6,7,8}$ as well as the response of bone health to interventions such as diet ${ }^{9,10}$, ovariectomy ${ }^{7,11}$ and pharmacologic agents ${ }^{8,12,13}$. BMD and bone micro-architecture at specific skeletal sites, namely the proximal tibia, femur and lumbar vertebrae, are indicative of overall bone health and of the risk of sustaining a fracture and so are the primary measures when quantifying responses to an intervention.

In vivo $\mu \mathrm{CT}$ image acquisition involves two-dimensional $\mathrm{X}$-ray projections being acquired at multiple angles as the $\mathrm{X}$-ray source and detector rotate around the animal under investigation ${ }^{14,15}$. The quality of the resulting image is dependent on many factors including, but not limited to: selected acquisition parameters (i.e., spatial resolution, X-ray voltage, amperage, rotation step, applied filter, exposure time), limitations of the $\mu C T$ scanner (i.e., scanner-based artifacts such as ring artifacts or dust that cause streaking or partial volume effects) and proper positioning and restraint of the animal. The former two of these factors can be manipulated to some degree by the user, depending on the specific scanning machine, study objectives and the corrections that are needed to optimize the function of the scanner or the processing of acquired images. The latter of these factors, the proper positioning of the rodent prior to scanning, can be achieved regardless of the scanner-based limitations or the acquisition parameters that are selected to achieve a specific study objective. While many publications involving in vivo imaging have been published in the literature ${ }^{14,15,16,17}$, classic manuscript style is such that detailed "how to" information cannot be included. Therefore, the aim of this article and video guide is to fill this void. Here we aim to instruct users of in vivo $\mu$ CT scanners how to anesthetize a rat, and position and restrain the hind limb to produce high quality images that can be analyzed to more accurately quantify outcomes of bone micro-architecture.

Preventing obstructions of the x-ray beam through objects other than the hind limb are imperative for quantifying the most accurate BMD and bone micro-architecture values. As the X-rays pass through objects and tissues of varying thickness and densities, some of the $\mathrm{X}$-rays are absorbed (i.e. attenuated) by the materials they pass through. Since the measured mass density of a sample is affected by its thickness, and the presence and thicknesses of surrounding tissues, it is imperative that calibration phantoms used to determine BMD are scanned in the same manner. Therefore, if the X-ray beam is to pass through objects (i.e., the tail) before or after passing through the region of interest, those objects will absorb some of the X-ray energies and will interfere with the transmission image acquired. In addition, these scans would be very difficult to 
simulate when scanning the phantoms that must closely resemble sample scans. As a result, these attenuation differences lead to inaccuracy in the evaluation of BMD measurements of the bone. Thus, for ease and accuracy, it is best to limit the number of obstructions between the $x$-ray source, region of interest and x-ray detector.

Longitudinal assessment of bone structure from an intervention in pre-clinical models involve the repeated anesthesia of the animal to limit their movement during scanning protocols. Several methods of general anesthesia exist in order to subdue the animals undergoing a $\mu \mathrm{CT}$ scan, including injectable and inhalant anesthesia ${ }^{1,2,4,5,6,12}$. Unlike inhalant anesthetics such as isoflurane, repeated general anesthesia using injectable anesthetics cause a reduction in body weight, surgical tolerance and significant changes to other physiological parameters in rodents, specifically rats and guinea pigs, suggesting significant contraindications for repeated use ${ }^{18,19,20}$. While isoflurane is highly volatile and allows for rapid induction and recovery, injectable anesthetic agents produce varying levels of anesthesia and time under anesthesia depends on the strain sex, body composition, fasted state, and circadian cycle of the animal. Injectable anesthetics also pose additional barriers to their use as they are highly regulated by national governing bodies. Inhalation anesthesia however, involves the direct delivery into the respiratory system; this method allows for faster induction and recovery time and better control over the length and depth of anesthesia ${ }^{19,20}$. Limitations to the inhalation anesthesia method involve its requirement for specialized vaporizing equipment and some changes to heart rate and blood pressure during induction, maintenance and recovery ${ }^{18,19}$.

\section{Protocol}

This study was approved by the Animal Care Committee of Brock University and conducted in accordance with the guidelines established by the Canadian Council on Animal Care ${ }^{21}$.

\section{Anesthesia Using Isoflurane Gas}

1. Pre-fill the acrylic glass incubation chamber with high-quality $\mathrm{O}_{2}$ at a continuous flow rate of approximately $1-2 \mathrm{~L} / \mathrm{min}$ from an anesthetic machine (Supplementary Figure 1).

2. Transfer the rat to the incubation chamber tail first and close the incubation chamber lid to create an airtight seal.

3. Begin to fill the incubation chamber with veterinary-grade isoflurane at $3-4 \% \mathrm{v} / \mathrm{v}$ dissolved in $\mathrm{O}_{2}$ at a continuous flow rate of $1-2 \mathrm{~L} / \mathrm{min}$ (Supplementary Figure 1).

CAUTION: Waste anesthetic gases can adversely affect handlers. A scavenger system (i.e., a charcoal filter or exhaust directly into a fume hood) must always be in place.

4. When the rat is no longer able to stand, transfer the rat to a face mask or nose cone receiving $1-3 \%$ isoflurane dissolved in $\mathrm{O}_{2}$ at a flow rate of 1-2 L/min. Rats primarily breathe through their nose and so, as long as the nose is covered by the face mask or nose cone, there will be sufficient anesthesia delivery.

5. Apply ophthalmic lubrication to the delicate membranes of the eyes to protect them from any escaped isoflurane gas. NOTE: Ensure that ophthalmic lubrication is without antibiotic, as this may affect outcomes from an intervention.

6. Measure palpebral (eye blinking response to gentle stimulation of palpebral opening) and pedal (withdrawal of hind limbs in response to pinching) reflexes; with increasing depth of anesthesia, palpebral reflex will be absent before pedal reflexes (Supplementary Figure 2).

7. When an appropriate level of anesthesia is reached and the rat has lost both palpebral and pedal reflexes, maintain the rat on $0.5-2 \%$ isoflurane dissolved in $\mathrm{O}_{2}$ at a flow rate of $1-2 \mathrm{~L} / \mathrm{min}$.

8. Continuously monitor the breathing rate of the rat throughout the procedure by keeping a constant visual on the rat either directly with an internal monitoring system or through the live-video feed (Supplementary Figure 3).

\section{Positioning and Restraint of the Rat Hind Limb}

1. Lay the rat in the supine position on the carbon-fiber scanner bed (Supplementary Figure 4).

2. Restrain the right foot in a malleable, foam tube, with the toes extending out of the end of the tube. Apply dental wax to hold the foot tightly within the foam and tape the tube closed tightly. Ensure that the diameter of the tube holding the foot is enough to fit firmly into the plastic tube.

3. Slide the plastic tube into the X-ray scanner bed (Supplementary Figure 5).

4. Extend the rat hind limb until it is taut. Do not over-extend the leg as to cause any harm to the rat (Supplementary Figure 5) as this may induce involuntary movement in the limbs due to labored respiration.

5. Pull the left leg (non-scanned hind limb) along with the tail out of the scanning field of view and toward the torso, away from the extended leg to be scanned.

6. Secure the left leg (non-scanned hind limb) and the tail in position using masking tape. Do not use anything more or less sticky (i.e., duct tape or painter's tape) as these materials will either harm the rat when they are removed (duct tape) or not provide a strong enough hold (painter's tape) (Supplementary Figure 6).

7. Secure the rat body into position at the hips, shoulders, and head with the masking tape. Secure the face mask or nose cone to the rat (Supplementary Figure 6).

NOTE: Blot the adhesive side of the masking tape to remove its ability to stick to the rat fur. Do not blot the ends of the masking tape so that it may be firmly secured to the scanning bed.

8. Wrap the rat in vet-wrap to limit heat loss (Supplementary Figure 6).

NOTE: When under general anesthesia, rats lose heat rapidly due to their large surface to body weight ratio ${ }^{19,20}$.

9. Continuously monitor the breathing rate of the rat throughout the procedure by keeping a constant visual on the rat (either directly or through a live-video feed).

NOTE: Here set up takes $5 \mathrm{~min}$, scan acquisition is acquisition setting-dependent, and recovery time is 60 mins.

10. Proceed to acquire the $\mu \mathrm{CT}$ images. 
NOTE: The exact specifications for scan acquisition are specific to each scanner type, software system and the specific research question, however, several methodological publications exist throughout the literature ${ }^{1,2,9}$.

\section{Recovery from Anesthesia}

1. After in vivo $\mu \mathrm{CT}$ scanning is complete, stop the flow of isoflurane to the rat but maintain a $1-2 \mathrm{~L} / \mathrm{min}$ flow of $\mathrm{O}_{2}$.

2. When the rat regains motor control (1-2 $\mathrm{min})$, remove it from the respirator and allow it to recover individually in a cage placed partially on a general-purpose heating pad at low heat. Rats are known to reduce their body temperature by $1^{\circ} \mathrm{C}$ when under general anesthetic ${ }^{19}$. Do not leave the rat unattended until it has regained sufficient consciousness to maintain sternal recumbency.

NOTE: Anecdotal evidence from our research group reports that immediately following recovery from isoflurane anesthesia, rats begin to eat and so it is important to have their food and water available to them during recovery. Although we have observed this behavior, repeated general anesthesia does not cause a significant increase in food intake or body weight ${ }^{1,9}$.

\section{Representative Results}

This method of anesthesia for the rat, and positioning and restraint of the hind limb for in vivo $\mu \mathrm{CT}$ imaging facilitates the acquisition of high quality images appropriate for the analysis of tibia micro-architecture. Proper positioning of the rat hind limb involves the leg being fully extended and the entire foot and ankle restrained in foam (Figure 1A) resulting in an acquired image of sufficient quality for analysis of the trabecular and cortical micro-architecture (Figure 1B). Insufficient placement and restraint of the hind limb (Figure 1C) can result in images with movement artifacts (Figure 1D), while a tail that is not fully removed from the scanning field of view (Figure 1E) will interfere with $X$-ray attenuation by the scanned samples (Figure 1F) and alter BMD and tissue mineral density (TMD) measurements. Either of these placement errors will result in a poor-quality scan that should not be further analyzed. Attaining images of poor quality will alter the quantification of the fine trabecular network and cortical structure of the hind limb and will produce inappropriate or inconclusive data ${ }^{14}$.
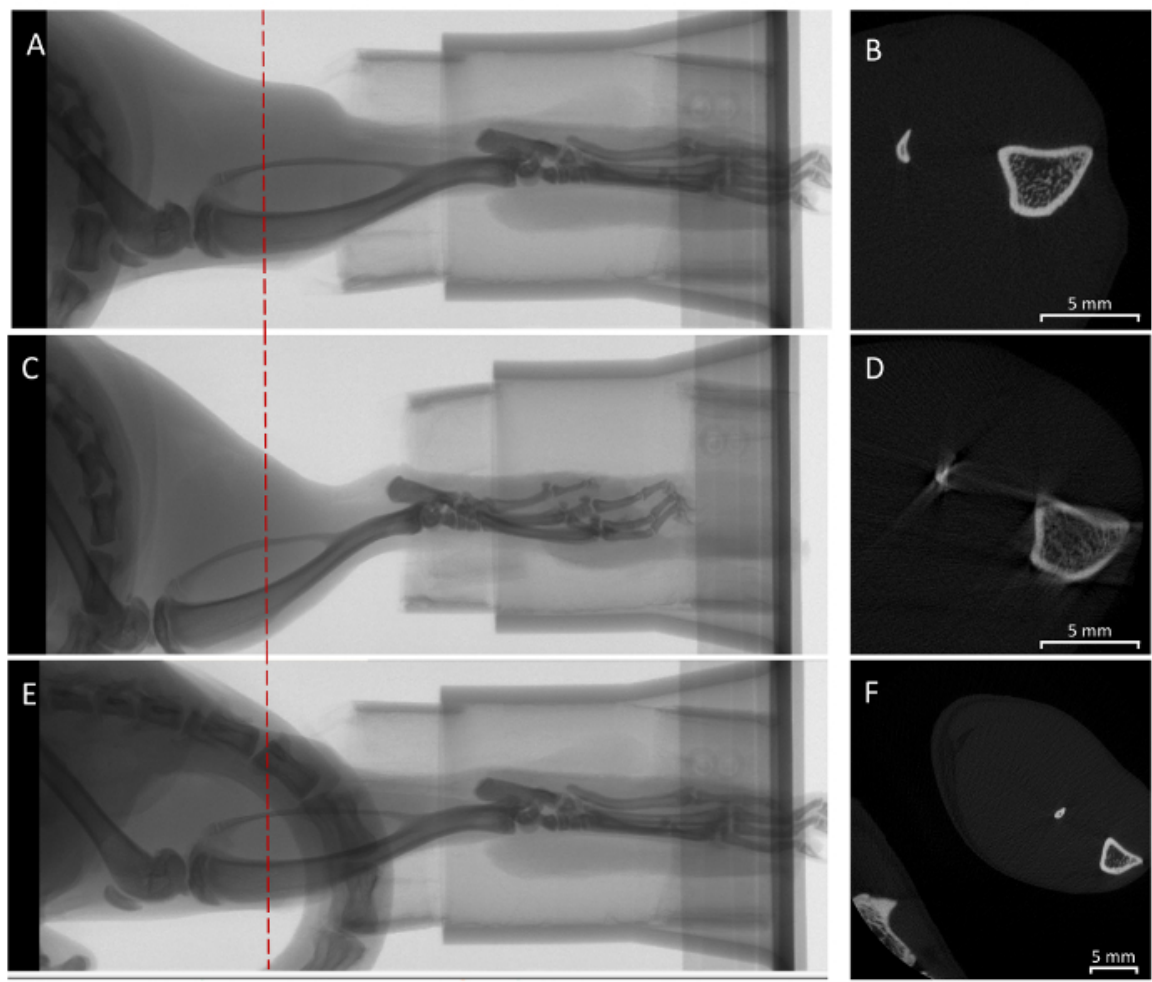

Figure 1. Representative images of placement of the rat hind limb and corresponding acquired images of the proximal tibia in crosssection.

(A) Proper placement of the rat hind limb with the ankle fully restrained in foam, leg extended and tail pulled away from the tibia provides sufficient image quality in (B) cross-section of the tibia and its trabecular and cortical micro-architecture. (C) Improper placement of the rat hind limb with the leg not fully extended and ankle not fully restrained in foam may result in (D) movement artifacts, seen as streaking in crosssection. (E) Objects interfering with the field of view, such as the tail not pulled away from the tibia $(F)$ interferes with $x$-ray attenuation from the tibia and can result in altered BMD and TMD measurements, although not visually evident. The bottom left corner in panel $\mathbf{F}$ shows a portion of tail in the field of view, that interfered with the x-ray beam that subsequently passed through the tibia. Red dotted lines in the left panels indicate the cross section presented on the right panels. Please click here to view a larger version of this figure.

Supplementary Figure 1. Isoflurane anesthetic unit. Isoflurane anesthetic unit set up to provide $3-4 \%$ isoflurane dissolved in $\mathrm{O}_{2}$ at a continuous flow-rate of 1-2 L/min for induction of general anesthesia. Please click here to download this figure. 
Supplementary Figure 2. Ensuring depth of anesthesia. Measure pedal reflexes by pinching the toes of the rat receiving continuous inhaled anesthetic through a face mask or nose cone. The pain response is more evident when the leg is slightly extended. Very strong pinches or the use of forceps or clamps can induce tissue damage and therefore should not be used. Please click here to download this figure.

Supplementary Figure 3. Screen capture of the live-feed physiological monitoring camera view. Please click here to download this figure.

Supplementary Figure 4. Rat laying in the supine position on the carbon-fiber scanner bed. Please click here to download this figure.

Supplementary Figure 5. Right foot of the rat restrained in a malleable foam tube. Right foot of the rat is restrained in a malleable foam tube with the toes extending out of the tube (not pictured here). The foam tube is restrained in a plastic holder (please refer to Table of Specific Materials/Equipment for more detailed information). Please click here to download this figure.

Supplementary Figure 6. Rat secured in position with the right leg extended straight. The tail and left foot is taped away from the right leg (toward the torso), the hips are secured and the rat torso is wrapped in vet-wrap (blue) to limit heat loss. Please click here to download this figure.

\section{Discussion}

This protocol provides viewers with the first detailed guideline for the proper anesthesia, placement, and restraint of the rat during in vivo $\mu \mathrm{CT}$ scanning of the hind limb. These guidelines enable users of in vivo $\mu$ CT scanning systems to obtain high resolution and high quality images of the tibia that can be processed for the quantification of 3-dimensional bone micro-architecture. Critical steps in the protocol necessary to ensure proper positioning and restraint involve the proper anesthesia of the rat as well as extending the hind limb away from all other critical structures until it is taut, but not in an unnatural position. For optimal imaging outcomes, it is imperative that the rat be anesthetized to a level of complete relaxation, and that palpebral and pedal reflexes are lost. In addition, the scanning leg should be extended and the entire foot and ankle should be restrained in foam. The methods described above to achieve optimal positioning of the scanning leg will ensure that: 1) hind limbs of rats within a study are consistently oriented in the same direction, thus permitting the $x$-ray beam to pass through the same area of each leg as it rotates around the sample; 2 ) both voluntary and involuntary movement of the hind limb will not occur, thus minimizing the potential for movement artifacts to interfere with the quality of acquired images; 3 ) obstructions from objects (i.e., the tail) are prevented, thus minimizing the potential for partial volume effects to produce inaccurate BMD and TMD measurements. These guidelines may be modified for each individual rat, as the rate of isoflurane metabolism and positioning can vary depending on strain and body size ${ }^{22}$. The most common in vivo scanning machines are designed for small animal models (i.e., mice, rats, rabbits, guinea pigs) and will have interchangeable animal stages to permit the scanning of different animal sizes. Therefore, they can accommodate a wide range of body weights.

Although in vivo $\mu \mathrm{CT}$ scanning permits for the rat to be repositioned and rescanned if the images acquired from the initial scan are poor quality, repeated scanning will expose the rat to additional doses of radiation and isoflurane anesthesia for a prolonged period of time. Monthly repeated radiation exposure of $600 \mathrm{mGy}$ focused to the rat tibia over four months does not cause adverse effects to bone micro-architecture compared to the contralateral hind limb ${ }^{1}$, but this does not ascertain the safety of two scans repeated in immediate succession. Further limitations of the technique described include the need to extend the hind limb taut with forces applied to it to keep it still, which may invoke some changes in bone structure. While the severity of restraint of the hind limb during scanning will depend on each research objective, previous research from our lab involving monthly repeated in vivo $\mu \mathrm{CT}$ imaging of one hind limb resulted in a difference in the cortical micro-architectural parameter, eccentricity, compared to the contralateral hind limb that did not undergo repeated extension, stabilization and scanning ${ }^{1}$. Eccentricity is a measure of the elliptical shape of the cortical bone and changes in response to altered load-bearing. Therefore, when using this method of positioning and restraining of the hind limb for repeated in vivo $\mu \mathrm{CT}$ imaging, consideration should be made when assessing and interpreting changes to load-bearing micro-architectural parameters.

While the above guidelines have been provided for the imaging and analysis of bone tissue, slight adjustments to the protocol must be made when imaging soft tissue of the hind limb. Specifically, the way in which the hind limb is extended from the torso and restrained must be taken into account, as the current procedure misshapes the orientation of the soft tissue (muscle, adipose tissue) into abnormal positioning for the duration of the scan. Therefore, when extrapolating this model for the use in imaging of soft tissue of the hind limb, some adjustments should be made to the restraint technique to reduce or eliminate the changes in the positioning of the tissues in relation to one another.

Furthermore, the guidelines have been written specifically based on the experiences of our research group, however, they can be modified to accommodate other commercially-available in vivo $\mu \mathrm{CT}$ scanners. Other suggested methods to position and restrain the hind limb may be available by the manufacturer of the in vivo $\mu \mathrm{CT}$ scanning system. Most commercially available in vivo $\mu \mathrm{CT}$ units list polypropylene, expanded polystyrene, and plastic tubes with dental wax to hold a protruding foot as acceptable materials and methods for restraining the scanning leg. However, the method presented in this protocol provide more controlled and consistent positioning and restraint of the scanned leg and, consistently produces high-quality images. The guidelines presented in the present method require specialized equipment necessary for the anesthesia of the rat, such as a vaporizer, tubes, masks, induction chambers, and oxygen. Although the equipment is associated with a somewhat higher cost compared to injectable anesthetics, it allows researchers the ability to rapidly and precisely induce anesthesia at specific depths of consciousness, which provides an advantage over alternative methods.

Using the guidelines outlined in the present method video, researchers utilizing high resolution in vivo $\mu \mathrm{CT}$ technologies to investigate their intervention of interest will be able to properly and consistently orient and restrain a rat hind limb for high quality X-ray imaging. This will provide a continuum in the field of in vivo $\mu \mathrm{CT}$ image acquisition and serve as a step towards optimizing the consistency and accuracy within studies and enable comparisons across studies in the literature. Similarly, these protocols and methods can be expanded for use in other rodent species, including mice, although some changes will be required ${ }^{2,10}$. For example, the restraint of the foot in the foam tube can include the ankle to minimize the possibility of leg movement during the scan. In addition, the full foot will fit into the foam holder. Thus, the toes do not extend out of the end of the holder as they do when securing the foot of a rat. In addition, the body of the mouse does not require the same restraint with tape as the rat. A smaller nose cone can be used for maintaining anesthesia in mice during the scan. If a smaller nose cone is not available, one can 
secure a nitrile glove over the available nose cone and make a small incision in the glove to provide a space that can fit the nose of the mouse to provide anesthesia while maintaining a seal around the nose.

While the proximal tibia is the main site of investigation of changes to bone micro-structure in the rat, guidelines for the proper and consistent positioning of other skeletal sites such as the femur and lumbar vertebrae should be investigated and established for consistency in the literature. However, when undertaking future research involving the imaging of the lumbar vertebrae, considerations must be made as imaging of the spine provides radiation exposure to the surrounding organs and tissues.

\section{Disclosures}

The authors have nothing to disclose.

\section{Acknowledgements}

The authors acknowledge research funding from a NSERC Discovery Grant (\#05573) and the Canada Foundation for Innovation (\#222084) for funding the in vivo micro-CT. W.E. Ward is a Canada Research Chair in Bone and Muscle Development.

\section{References}

1. Longo, A. B., Sacco, S. M., Salmon, P. L., \& Ward, W. E. Longitudinal use of micro-computed tomography does not alter microarchitecture of the proximal Tibia in sham or ovariectomized sprague-dawley rats. Calcif Tissue Int. 98 (6), 631-641 (2016).

2. Sacco, S. M. et al. Repeated irradiation from micro-computed tomography scanning at 2, 4 and 6 months of age does not induce damage to tibial bone microstructure in male and female CD-1 mice. Bonekey Rep. 6855 (2017).

3. Waarsing, J. H., Day, J. S., Verhaar, J. A., Ederveen, A. G., \& Weinans, H. Bone loss dynamics result in trabecular alignment in aging and ovariectomized rats. J Orthop Res. 24 (5), 926-935 (2006).

4. Klinck, R. J., Campbell, G. M., \& Boyd, S. K. Radiation effects on bone architecture in mice and rats resulting from in vivo micro-computed tomography scanning. Med Eng Phys. 30 (7), 888-895 (2008).

5. Laperre, K. et al. Development of micro-CT protocols for in vivo follow-up of mouse bone architecture without major radiation side effects. Bone. 49 (4), 613-622 (2011).

6. Brouwers, J. E., van Rietbergen, B., \& Huiskes, R. No effects of in vivo micro-CT radiation on structural parameters and bone marrow cells in proximal tibia of wistar rats detected after eight weekly scans. J Orthop Res. 25 (10), 1325-1332 (2007).

7. Francisco, J. I., Yu, Y., Oliver, R. A., \& Walsh, W. R. Relationship between age, skeletal site, and time post-ovariectomy on bone mineral and trabecular microarchitecture in rats. $J$ Orthop Res. 29 (2), 189-196 (2011).

8. Altman, A. R. et al. Quantification of skeletal growth, modeling, and remodeling by in vivo micro computed tomography. Bone. $81370-379$ (2015).

9. Longo, A. B. et al. Lifelong intake of flaxseed or menhaden oil to provide varying n-6 to n-3 PUFA ratios modulate bone microarchitecture during growth, but not after OVX in Sprague-Dawley rats. Mol Nutr Food Res. 61 (8), (2017).

10. Sacco, S. M., Saint, C., LeBlanc, P. J., \& Ward, W. E. Maternal consumption of hesperidin and naringin flavanones exerts transient effects to tibia bone structure in female CD-1 offspring. Nutrients. 9 (3),250 (2017).

11. Campbell, G. M., Buie, H. R., \& Boyd, S. K. Signs of irreversible architectural changes occur early in the development of experimental osteoporosis as assessed by in vivo micro-CT. Osteoporos Int. 19 (10), 1409-1419 (2008).

12. De Schaepdrijver, L., Delille, P., Geys, H., Boehringer-Shahidi, C., \& Vanhove, C. In vivo longitudinal micro-CT study of bent long limb bones in rat offspring. Reprod Toxicol. 46 91-97 (2014).

13. Perilli, E. et al. Detecting early bone changes using in vivo micro-CT in ovariectomized, zoledronic acid-treated, and sham-operated rats. Osteoporos Int. 21 (8), 1371-1382 (2010).

14. Bouxsein, M. L. et al. Guidelines for assessment of bone microstructure in rodents using micro-computed tomography. J Bone Miner Res. 25 (7), 1468-1486 (2010)

15. Li, H., Zhang, H., Tang, Z., \& Hu, G. Micro-computed tomography for small animal imaging: Technological details. Progress in Natural Science. 18 (5), 513-521 (2008)

16. Campbell, G. M., \& Sophocleous, A. Quantitative analysis of bone and soft tissue by micro-computed tomography: applications to ex vivo and in vivo studies. Bonekey Rep. 3564 (2014).

17. Meganck, J. A., Kozloff, K. M., Thornton, M. M., Broski, S. M., \& Goldstein, S. A. Beam hardening artifacts in micro-computed tomography scanning can be reduced by X-ray beam filtration and the resulting images can be used to accurately measure BMD. Bone. 45 (6), 1104-1116 (2009).

18. Vazquez, C. M., Molina, M. T., \& llundain, A. Role of rat large intestine in reducing diarrhea after $50 \%$ or $80 \%$ distal small bowel resection. Dig Dis Sci. 34 (11), 1713-1719 (1989).

19. Albrecht, M., Henke, J., Tacke, S., Markert, M., \& Guth, B. Effects of isoflurane, ketamine-xylazine and a combination of medetomidine, midazolam and fentanyl on physiological variables continuously measured by telemetry in Wistar rats. BMC Vet Res. 10 198 (2014).

20. Schmitz, S., Tacke, S., Guth, B., \& Henke, J. Comparison of physiological parameters and anaesthesia specific observations during isoflurane, ketamine-xylazine or medetomidine-midazolam-fentanyl anaesthesia in male guinea pigs. PLoS One. 11 (9), e0161258 (2016)

21. Canadian Council on Animal Care. Guide to the care and use of experimental animals. <http://www.ccac.ca/Documents/Standards/ Guidelines/Experimental_Animals_Vol1.pdf> (1993).

22. Stevens, W. C. et al. Comparative toxicities of halothane, isoflurane, and diethyl ether at subanesthetic concentrations in laboratory animals Anesthesiology. 42 (4), 408-419 (1975). 